\title{
Sense Logic
}

\author{
(part 1) \\ Fundamental Concept
}

[P-S Standard]

Egger Mielberg

egger.mielberg@gmail.com

01.07.2021

\begin{abstract}
.
Traditional mathematical logic is "what follows from what".

Sense logic - "what belongs to what".

Traditional mathematical logic is "a collection of abstract objects not related to the outside world."

Sense logic is "a set of objects and events that describe the state of the real world."

Below, we present a new paradigm of logic based on semantic connections between the considered objects of any nature. The Sense Logic is not a part of traditional mathematics. Its main task is to describe the phenomena of the real world from the standpoint of their semantic coherence.
\end{abstract}

\section{Introduction}

In traditional mathematics, $a \in Z, a=-1, N \subseteq Z$, respectively, $a=-1 \rightarrow$ $a \notin N$. 
In Sense Theory [1], $b \in O_{N}, O_{N} \in{ }^{\Phi_{\text {oN }}},{ }^{\Phi_{\text {oN }}} \subseteq S_{c}$. Further, according to the rules of mathematical logic, we should get the following expression:

$$
b \in \Phi^{\Phi_{(\mathbb{N})}}
$$

However, it is not true since $b \notin S_{c}\left(\lim O_{N} \neq S_{c}\left(Z_{0}\right)\right)$.

Also, unlike the Category Theory, which studies the properties of relations between mathematical objects by operating with sets of morphisms between them, the Sense Space [2] as well as the Sense Function [3] is based on the concept of direct \& reverse surjection. In addition, the problem of extending the concept of "class" to more arbitrary "classes" as elements of another arbitrary set is usually solved through the introduction of the axiom of the existence of the Grothendieck universe. Grothendieck's universe, in turn, is completely abstracted from the properties of the set elements with which it operates.

In mathematical logic, for example, the concept of belonging of any element $x_{i}$ to a certain set $X_{N}$ describes a formal attribute of a relationship:

$$
x_{i} \in X_{N} \text {, where } X_{N}=\left\{x_{1}, x_{2}, x_{3}, \ldots, x_{n}\right\},
$$

where $x_{1}$ has the same meaning in the sense of "belonging to the set $X_{N}$ " as other elements of this set. For example, the set of natural numbers $\mathrm{N}$ unites its elements on the basis of the "non-negativity" of their values, as well as countability.

However, it does not say anything about the influence of each individual element both on the set itself and on its other elements. Also, the result of the intersection of two natural sets $\mathrm{N}$ and $\mathrm{M}$ is not obvious in terms of the influence of replacing one or all elements of this intersection on the properties of these two sets.

In semantic theory, the concept of belonging of any element $a_{1}$ to a certain set $A_{N}$ describes a quantitative or qualitative 
attribute of a relationship to an object $Z_{0}$, which is common for all elements of a given set:

$$
a_{1} \underset{\mathrm{S}}{\in} A_{N} \rightarrow \exists \mathrm{S}\left(\bigcirc \odot A_{N}=S\right)
$$

(in terms of the implication of mathematical logic)

$$
a_{1} \in \underset{S}{\in} A_{N} \stackrel{\text { or }}{\rightarrow} \lim _{\mathrm{S}} A_{N} \neq \emptyset_{S}
$$

(in terms of the semantic implication of the Sense Theory)

\section{Problem}

In classical mathematical logic, logical statements define affirmative sentences about which you can judge whether they are true or false. That is, sentences that lend themselves to analysis from the point of view of either their falsity or their truth, the third is not given.

In the Sense Logic, logical statements define any kind of sentence - interrogative, exclamatory, affirmative, imperative, and so on.

That is, sentences in which there is a semantic link, a sense limit [1] between the words of one sentence or several sentences. In terms of the Sense Theory, there must be sense direct surjection [2] between the words of one sentence or the words of two or more sentences under consideration. This approach allows us to consider even any meaningless statements that, in traditional mathematical logic, do not lend themselves to any analysis. 
The main problem in creating a self-learning $\mathrm{Al}$ is the absence of any theory (mathematical, physical) that works in practice that would not divide the world into black and white (true or false), but would allow us to consider an object or event of any nature from the position of their coherence and influence on each other.

Below we present a new paradigm of "logic" in the field of working with data objects of large and extremely large volumes, as well as events associated with these objects by "meaning".

\section{Solution}

The basic concepts of the Sense Logic are sense sentences and semantic operations on these sentences.

A sense sentence is any sequence of words, phrases, phrases and sentences that belongs to at least one object (zero object) of different nature, describing its properties or its state, or both. In other words, this sequence unites all of its elements with one meaning, a sense object.

An example of a sense sentence that does not explicitly contain a sense object:

"Anyone who reads this text can read."

The sense object in this sentence can be any animate or inanimate creature (man or computer).

An example of a sense sentence that does explicitly contain a sense object:

"The seatbelt slammed into the pilot's shoulders as the speed increased rapidly."

The object in this sentence is the pilot.

Sentences that do not contain a description of the properties or states of any object are not sense sentences. For example: 
"Success is achieved through daily hard work."

The object "success" in this sentence does not contain a description of its state or any of its properties. However, this object has a semantic relationship to the object "work" (see "Logic of Sense Relations").

The overwhelming majority of definition sentences are sense sentences:

"Water is an inorganic chemical substance, which is the main constituent of Earth's hydrosphere..."

In the Sense Logic there are a number of basic semantic operations that make it possible to determine the "meaning" and the content of the objects under consideration included in one or more sentences, as well as the semantic connection between them. Among them:

1. Semantic Union

2. Semantic Intersection

3. Semantic Subset

For more details, see Appendix.

\section{Semantic Union}

$A$ semantic union of two sentences $A$ and $B$, each consisting of one or more objects, is a sentence $C$ that satisfies the following condition:

$$
\left.\forall c \in C\left(c_{i} \in \underset{\mathrm{S}}{\in} \boldsymbol{\phi}_{\mathrm{M}} \mid \boldsymbol{\phi}_{\mathrm{M}}=\biguplus\right) \boldsymbol{\phi}_{\mathrm{K}}, \boldsymbol{\phi}_{\mathrm{L}}, \boldsymbol{\phi}_{\mathrm{K}} \subseteq A, \boldsymbol{S}_{\mathrm{L}} \subseteq B\right)
$$


Union Sense Table

\begin{tabular}{|l|l|l|}
\hline$O_{M}$ & $\not_{\mathrm{K}}$ & $\boldsymbol{\phi}_{\mathrm{L}}$ \\
\hline $\boldsymbol{S}_{\mathrm{L}}$ & $\not{\phi}_{\mathrm{M}}$ & $\boldsymbol{\phi}_{\mathrm{L}}$ \\
\hline $\boldsymbol{\phi}_{\mathrm{K}}$ & $\boldsymbol{\phi}_{\mathrm{K}}$ & $\boldsymbol{\phi}_{\mathrm{M}}$ \\
\hline
\end{tabular}

In the Union Sense Table, sentences (other objects) for which there is at least one zero object $Z_{0}$ are always located diagonally.

Semantic Intersection

A semantic intersection of two sentences $A_{K}$ and $B_{L}$, each consisting of one or more objects, is a sentence $C$ that satisfies the following condition:

$$
\forall c \in C\left(c_{K}=a_{i}, c_{K}=b_{j} \mid\left\{a_{i}\right\} \stackrel{\mathrm{S}}{=}\left\{b_{j}\right\} \text { for } i, j=\{1,2,3, \ldots n\} \text { where } n \leq K, L\right)
$$

Intersection Sense Table

\begin{tabular}{|l|l|l|l|l|}
\hline$O_{K}$ & $a_{1}$ & $a_{2}$ & $a_{3}$ & $\cdots$ \\
\hline$b_{1}$ & $c_{1}$ & & & \\
\hline$b_{2}$ & & $c_{2}$ & & \\
\hline$b_{3}$ & & & $\cdots$ & \\
\hline$\cdots$ & & & & $c_{K}$ \\
\hline
\end{tabular}

In the Intersection Sense Table, sentences (other objects) that form a sense sequence converging to at least one zero object $O_{K}$ are always located diagonally. 
Semantic Subset

A semantic subset of sentence $A_{N}$ is a sentence $B_{K}$ that satisfies the following condition:

$$
\lim _{S} B_{K} \in \boldsymbol{S}_{\mathrm{N}}\left(A_{N}\right)
$$

provided that both sense limits $\lim _{S} A_{N}$ and $\lim _{S} B_{K}$ exist.

Subset Sense Table

\begin{tabular}{|c|c|c|c|c|}
\hline K N & $a_{1}$ & $a_{2}$ & $\cdots$ & $a_{n}$ \\
\hline$b_{1}$ & $\begin{array}{c}O_{K}=a_{1} \\
\text { or }\end{array}$ & & & \\
\hline$b_{2}$ & & $\begin{array}{c}O_{K}=a_{2} \\
\text { or }\end{array}$ & & \\
\hline$\cdots$ & & & $\cdots$ & \\
\hline$b_{k}$ & & & & $O_{K}=a_{n}$ \\
\hline
\end{tabular}

In the Subset Sense Table there is a single value-object (in the case of uniqueness of sentence $B_{K}$ ) that stands on the diagonal and is the sense limit of $B_{K}$.

In the Sense Logic there are a number of additional semantic operations that allow describing semantic interaction and the relationship between whole sentences or paragraphs of sentences.

The Sense Logic is divided into two main sections - "Logic of Senses" and "Logic of Sense Relations". 


\section{Formulas for the Logic of Senses}

The elements of a sense sentence are letters, special characters or symbols, words, phrases and sentences containing alphanumeric information.

Semantic formulas are built using elements of sense sentences, semantic operations and brackets that determine the order of semantic sequence.

Unlike traditional logic, where the induction method or the inductive method is actively used to obtain "new" knowledge about the phenomenon under study, in the Sense Logic as well as in the Sense Theory in general, this method is not applicable. The main reason for the inapplicability of this method lies in its limitation on the existence of one common feature in the entire general sample of individual phenomena.

This limitation is a very weak criterion for generalizing the sense component of a small sample of phenomena to its larger set.

For example, the set $A$ of people consists of elements $A_{1}, A_{2}$, $A_{3}, \ldots, A_{n}$, where the main feature of the uniting people in the set $A$ is the presence of brains in the physical sense. Further, using the method of full induction, we conclude that all people without exception who have a chemical in their skull called brains can be attributed to set $A$. Now let's look at two real-life situations. In the first situation, we assign a natural number $\mathrm{N}$ to a chemical substance of an individual person, reflecting the number of cells and the bonds between them of a given substance. In the second situation, each of the elements $A_{i}$ will be assigned the number 2, reflecting the presence of two eyes. Further, over time, the following two facts become apparent:

1. The number $\mathrm{N}$ will be radically different from each other for each of the elements of $A_{i}$. 
2. The number 2 with a certain probability may not exist for individual elements of $A_{i}$.

In other words, the classical inductive approach works well when using static features, and is not at all applicable for features that change their meaning over time. Therefore, the classical inductive approach is severely limited in its application to natural phenomena.

The Logic of Senses, as well as the Sense Theory as a whole, use their own approach both, in obtaining "new" knowledge and generalizing the obtained knowledge of a small sample to a more general sample.

\section{"Sense-In" method}

This method uses a similar paradigm of the transition "from the general to the particular" implemented in the deductive method. However, the semantic component $Z_{0}$ (zero object) is used as a key sign of "transition" in the Sense-In method. So using the above example to find one local sample with a common feature, say the presence of education, we can use semantic integral $S_{f}\left(A_{i}\right)$ on $p_{1}$ on union, where $p_{1}$ is the education [4]:

$$
\oint_{p_{i}}^{\circ}\left[S_{f}\left(A_{i}\right)\right]=\underset{\ominus}{\operatorname{diff} f}\left(p_{1}\right)\left[S_{f}\left(A_{i}\right)\right]_{L}=S_{f}\left(A_{i-L}\right)
$$

where $p_{1}$ - first property of $A_{i}, p_{1} \notin A_{L}$.

Moreover, the semantic integral can be calculated the number of times that the researcher needs to obtain, for example, a limited sample from $A$ with a limited number of features. It is worth noting that already the first integral will filter out only those elements from $A$ that have the "education" property. 
If necessary, the "transition" from the general set of properties $A$ to some separately taken properties of this set can be realized through the application of semantic integral on $p_{i}$ on union, where $i=\{1,2,3, \ldots n\}$.

Thus, the Sense-In method makes it possible to move from a general sample of a set of a certain nature with a certain set of properties to a local "by sense" sample with a limited set of properties or one property. The practical advantage of this method is its ability to work with dynamic properties.

For the problems of studying additional properties over all elements of the set $A_{i}$, the semantic integral on object [4] can be used.

\section{"Sense-Out" method}

This method uses a similar paradigm of the transition "from the particular to the general" implemented in the inductive method.

However, as in the Sense-In method, the semantic component $Z_{0}$ (zero object) is used as a key sign of "transition" in the Sense-Out method. So using the above example to find one general sample common for all elements of the set $A_{i}$ with common properties, we can use the semantic integral $S_{f}(A)$ on $A_{i}$ on disunion [4]:

$$
\oint_{A}^{\ominus}\left[S_{f}(A)\right]=\underset{b}{\operatorname{dif} f} f\left(A_{i}\right)\left[S_{f}(A)\right]_{M}=S_{f}\left(A_{i+M}\right)=A_{i}=\text { const }
$$

That is, the semantic integral on object allows one to obtain additional information on additional "new" properties of an individual element of the set $A_{i}$ that the entire general sample $A$ possesses. 
Moreover, an increase in the number of elements $A$ can be realized without losing the basic or "genetic" properties of the set $A_{i}$.

The scheme for describing the semantic connections between theory, practice (empiricism), the Sense-In method and the Sense-Out method can be represented as follows:

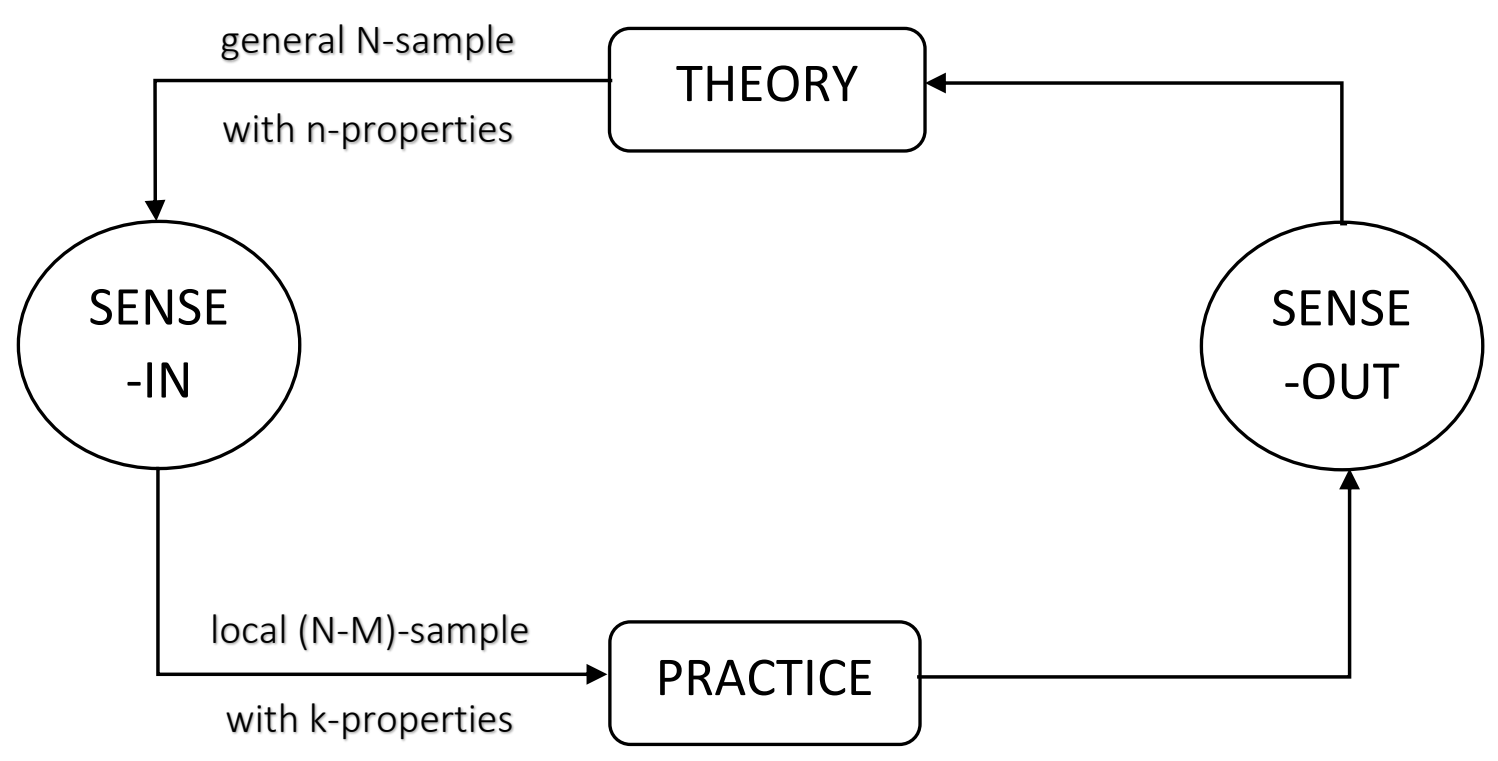

Depending on the tasks assigned to the researcher, the value of $k$ can be either less or equal to the value of $n$. So, for example, when setting the task of studying the "genetic" properties of an individual element $A_{i}$, the scheme for representing semantic connections will be as follows: 


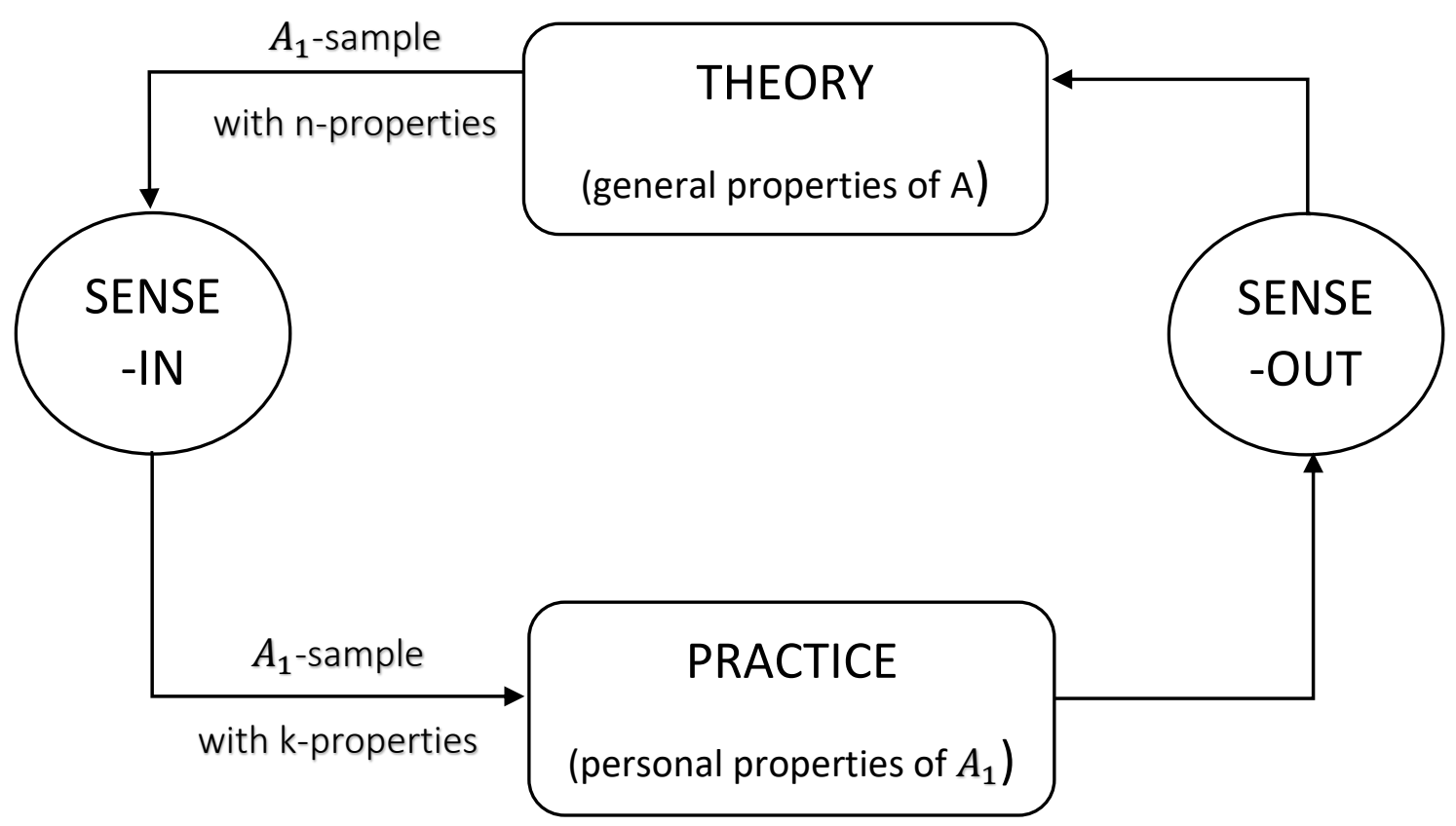

where $k \ll n$.

The formulas for the Logic of Senses are defined according to the following several rules:

1. Any expression consisting of at least one element of a sense sentence and one semantic operation is a formula.

2. If $A$ and $B$ are formulas, then expressions composed using $A, B$ and semantic operations will also be formulas.

3. If a set $\{A\}$ of any dimension and of any nature is not a formula, then if it is possible to add one semantic operation between the elements of this set, it becomes a formula.

As in the case of classical logic, brackets in the Sense Logic can be omitted in some situations. However, in this case, the order of precedence of semantic operations must be observed:

$$
\odot-\odot,(\ominus)-(\ominus), \text { ( }-\stackrel{s}{\complement}
$$




\section{Formulas for the Logic of Sense Relations}

Unlike the formulas of the Logic of Senses, where the main focus in their construction is the identification of the meaning of objects of one or a set of sentences, the formulas of the Logic of Sense Relations describe the possible existence or absence of a semantic connection between the objects under consideration or whole sentences.

The formulas for the Logic of Sense Relations are defined according to the following several rules:

1. Any expression consisting of at least one element of a sense sentence and one of four binary operations -

$$
\text { ค, } \stackrel{s}{\complement}, \stackrel{s}{\rightarrow}, \stackrel{s}{=}
$$

is a formula.

2. If $A$ and $B$ are formulas of the Logic of Sense Relations, then expressions composed using A, B and semantic

$$
\text { ค, } \stackrel{s}{\complement}, \stackrel{s}{\rightarrow}, \stackrel{s}{=}
$$

operations

will also be formulas.

3. Any formula of the Logic of Sense Relations consisting of operands with more than one element of a sense sentence can be split into two or more formulas.

Formulas of the Logic of Senses and the Logic of Sense Relations are called semantic formulas.

\section{Sense Functions}

Unlike classical mathematics, where each logical formula takes only one of two values, 0 (false) or 1 (true), in the Sense Theory formulas can contain more than one sense (zero object) in each of their operands. Thus, the sense function $S_{f}[3]$ is strictly surjective.

Any sense function $S_{f}$ can be set in three ways: 


\section{Analytically}

2. Graphically

3. Tabularly

One of the forms of the table representation of the function $S_{f}$ can be as follows:

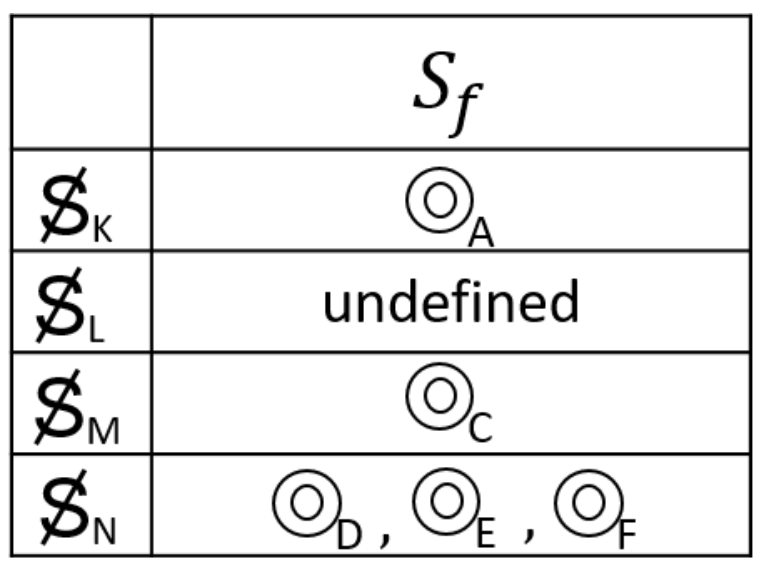

The number of all possible n-dimensional No-Sense Sets defining the function $S_{f}$ is equal to the sense number $S_{N}$ of these sets.

Definition 1:

The sense number $S_{N}$ of the set $A$ is the number of combinations of elements of the set $A$, each of which has its limit at least one zero object.

\section{Definition 2:}

The power $P_{S}$ of a Complete Sense Set $S_{C}$ is equal to or less than its sense number:

$$
P_{S} \leq S_{N}
$$

due to the possibility of the existence of the following expression:

$$
S_{f}(A) \stackrel{s}{\neq} S_{f}(A)
$$


Any $S_{f}$ can be specified using a semantic formula.

The binary operation $\subset$ ("semantic inclusion") plays a special role in the Sense Theory. This operation naturally interprets the presence of a semantic connection between an arbitrary object $\left(Z_{0}\right)$ of arbitrary nature and an arbitrary set (No-Sense Set) describing it.

So, for example, the formula

$$
\left(A_{K} \text { ๒ } B_{L}\right) \odot C_{M}
$$

defines a Sense Set $S$ only if the expression $\left(A_{K}\right.$ b) $\left.B_{L}\right)$ defines a sense function $S_{f}$.

\section{Conclusion}

In this article, we have presented an initial description of the Sense Logic based on the fundamental principles of the Sense Theory. We believe that a radically new approach with new tools for analyzing big data will help us get closer to understanding the task of building a full-fledged self-learning Al. We hope that our decent work will help other Al researchers in their life endeavors.

To be continued. 


\section{Appendix}

\section{Part 1.}

$\subsetneq$ - "inclusion", binary operation

1. $\mathrm{O}_{\mathrm{f}(1)} \odot \mathrm{O}_{\mathrm{p}(1)}=\mathrm{O}_{\mathrm{p}(1)} \odot \mathrm{O}_{\mathrm{f}(1)} \quad$ (“O” commutativity)

2. (0) $\odot \mathscr{S}=\mathscr{S} \odot($ () ("O/NS" commutativity)

3. $\mathrm{S}_{\mathrm{f}(\mathrm{N})} \odot \mathrm{O}_{\mathrm{p}} \neq \mathrm{O}_{\mathrm{p}} \odot \mathrm{S}_{\mathrm{f}(\mathrm{N})} \quad$ ("S/O" inequality)

\section{Operation that does not make a sense:}

1. $\Phi_{M} \subset \Phi_{L}$

2. $\$ \subset \mathrm{O}_{\mathrm{N}}$ or $\mathrm{O}_{\mathrm{N}} \subset \not$

3. $($ ) $\subset S$ or $S \subset($

4. $\$ \subset S$ or $S \subset \not$

5. () $\subset \mathrm{O}_{\mathrm{N}}$ or $\mathrm{O}_{\mathrm{N}} \subset(0$

6. () $\subset$ ()

(O) - "semantic union", binary operation

1. $\mathscr{S}_{\mathrm{M}}(\rho) \mathscr{S}_{\mathrm{L}}=\mathscr{S}_{\mathrm{L}}(0) \mathscr{S}_{\mathrm{M}}$ ("NS" commutativity)

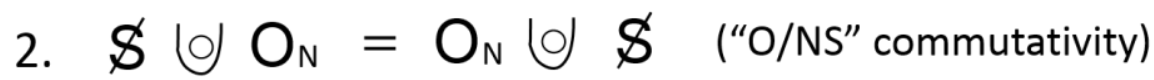

3. $\bigcirc_{A}(O) \bigcirc_{B} \neq \bigcirc_{B}(O) \bigcirc_{A}$

4. $\mathrm{O}_{\mathrm{M}}\left(\mathrm{O}_{\llcorner} \neq \mathrm{O}_{\llcorner} \odot \mathrm{O}_{\mathrm{M}}\right.$

5. $S_{M}\left(O_{\llcorner} \neq O_{\llcorner} \bullet S_{M}\right.$ 
Operation that does not make a sense:

1. (a) () $\$$ or $\$(0)$

$\ominus$ - "exclusion", binary operation

1. $\mathrm{O}_{\mathrm{fp}(2)} \odot \mathrm{O}_{\mathrm{p}(1)} \neq \mathrm{O}_{\mathrm{pf}(2)} \odot \mathrm{O}_{\mathrm{f}(1)}$

2. $\odot \odot \not \neq \not \subset \odot()$

3. $\mathrm{S}_{\mathrm{fp}(\mathrm{N}+1)} \odot \mathrm{O}_{\mathrm{p}} \neq \mathrm{O}_{\mathrm{p}} \subset \mathrm{S}_{\mathrm{fp}(\mathrm{N}+1)}$

Operation that does not make a sense:

1. $\phi_{2(\mathrm{M})} \odot \phi_{1(\mathrm{~L})}$

2. $\not \subset \mathrm{O}_{\mathrm{N}}$ or $\mathrm{O}_{\mathrm{N}} \odot \not$

3. $\odot \odot S$ or $S \odot(0$

4. $\$ \odot S$ or $S \odot \phi$

5. $\odot \odot \mathrm{O}_{\mathrm{N}}$ or $\mathrm{O}_{\mathrm{N}} \odot($

6. (0) $\odot \odot$

$\ominus$ - "semantic disunion", binary operation

1. $\mathscr{S}_{\mathrm{N}} \ominus \mathscr{S}_{\mathrm{L}} \neq \mathscr{S}_{\mathrm{L}} \ominus \mathscr{S}_{\mathrm{N}}$

2. $\Phi_{N}(\ominus) O_{K} \neq O_{K}(\ominus) \Phi_{N}$

3. $\bigcirc_{1(2)} \ominus \bigcirc_{1} \neq \bigcirc_{1} \biguplus \ominus \bigcirc_{1(2)}$

4. $O_{\mathrm{N}} \ominus \mathrm{O}_{\llcorner} \neq \mathrm{O}_{\llcorner} \ominus \mathrm{O}_{\mathrm{N}}$

5. $S_{N} \ominus O_{K} \neq O_{K} \ominus S_{N}$ 
Operation that does not make a sense:

1. (†) $\ominus$ or $\$(\ominus)$

O) - "semantic intersection", binary operation

1. $\mathscr{S}_{\mathrm{N}} \bigcirc \mathrm{S}_{\mathrm{N}}($ it. 2$)$

2. $\Phi_{N} \bigcirc O_{N}$ if and only if $\Phi_{N}|\stackrel{E}{E} \Rightarrow| S_{N}\left(O_{N}\right)$

3. $\bigcirc_{1(\mathrm{M})} \bigcirc \bigcirc_{1(\mathrm{~L})}$ if and only if $\bigcirc_{1(\mathrm{M})} \mid \mathrm{E}=>\bigcirc_{1(\mathrm{~L})}$

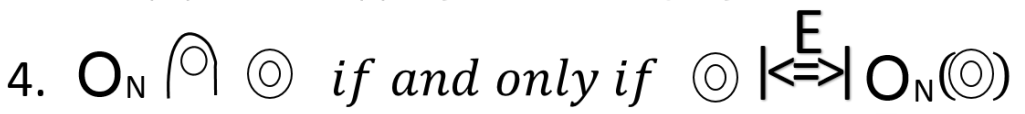

5. $S_{N} \cap(0)$ if and only if $S_{N}(0)|K=|$

6. $\mathrm{O}_{\mathrm{N}} \bigcirc \mathrm{O}_{\mathrm{K}}$ if and only if $\mathrm{O}_{\mathrm{N}}(\mathrm{O}) \mathrm{K} \stackrel{\mathrm{E}}{=}=1 \mathrm{O}_{\mathrm{K}}(\mathrm{O})$

7. $\mathrm{O}_{\mathrm{N}} \bigcirc \mathrm{S}_{\mathrm{K}}(i t .6)$

8. $\mathscr{S}_{\mathrm{N}} \cap \mathscr{S}_{\mathrm{K}}$ if and only if $\mathscr{S}_{\mathrm{N}} \mid \stackrel{\mathrm{E}}{=}>\mathscr{S}_{\mathrm{K}}$

Operation that does not make a sense:

1. () $\$$ or $\$$ ค (

"Semantic Intersection" is commutative for all operands.

$S$

- "semantic subset", binary operation

1. $\mathrm{S}_{\mathrm{K}} \stackrel{\mathrm{S}}{\complement} \mathrm{S}_{\mathrm{N}}$ if $\mathrm{O}_{\mathrm{K}} \in \mathscr{S}_{\mathrm{O}(\mathrm{N})}$

2. $\mathrm{O}_{\mathrm{M}} \stackrel{\mathrm{S}}{\complement} \mathrm{O}_{\mathrm{L}}$ if $\mathrm{O}_{\mathrm{M}} \in \mathscr{S}_{\mathrm{O}(\mathrm{L})}$ 
1. $\mathrm{S}_{\mathrm{K}} \stackrel{\mathrm{S}}{\not} \mathrm{S}_{\mathrm{N}}$ if $\mathrm{O}_{\mathrm{K}} \notin \mathscr{S}_{\mathrm{O}(\mathrm{N})}$

2. $\mathrm{O}_{M} \quad \mathrm{O}_{L}$ if $\mathrm{O}_{\mathrm{M}} \notin \mathscr{S}_{\mathrm{O}(\mathrm{L})}$

$|<\stackrel{\text { E}}{=}|$ - "equivalence", binary operation

Object $O_{N}$ is equivalent to Object $O_{K}$ if the following two conditions are met:

1. length $\left(\boldsymbol{\phi}_{(\mathrm{N})}\right)=$ length $\left(\boldsymbol{\phi}_{(\mathrm{K})}\right)$.

2. $\mathrm{O}_{\mathrm{N}}(\bigcirc) \in \mathcal{S}_{\mathrm{K}}$ and $\mathrm{O}_{\mathrm{K}}(\bigcirc) \in \mathcal{S}_{\mathrm{N}}($ can be $)$

$\stackrel{S_{1}}{\longrightarrow}$ - "semantic connection", binary operation

Definition 9: Object A semantically connects to Object B if the following expression is true:

$$
\$(A)_{N} \underset{1<=>\mid}{E} S(B)_{N}
$$

"Semantic connection" (SC) - is measured by percent. The following formula is used:

$$
S C_{\%}=\frac{N_{S} * 100}{N_{M}}
$$

where $N_{S}$ - number of similar properties of both objects, $N_{M}$ - number of properties of largest object. 
$\underline{\mathrm{S}}$ - "semantic negation", unary operation

$\mathrm{S}_{\neg} O_{N}$ means any Object $O_{K}$ that satisfies the following condition:

$$
\mathrm{O}_{\mathrm{K}}|\stackrel{\not E}{=}>| \mathrm{O}_{\mathrm{N}}
$$

Associativity ("inclusion"):

$$
\left(\mathrm{O}_{\mathrm{A}(1)} \odot \mathrm{O}_{\mathrm{B}(1)}\right) \odot \mathrm{O}_{\mathrm{C}(1)}=\mathrm{O}_{\mathrm{A}(1)} \odot\left(\mathrm{O}_{\mathrm{B}(1)} \odot \mathrm{O}_{\mathrm{C}(1)}\right)
$$

Associativity ("semantic union"):
$\left(\mathrm{O}_{A}(0) \mathrm{O}_{B}\right)(O) \mathrm{O}_{C}=\mathrm{O}_{A}(O)\left(\mathrm{O}_{B}(O) \mathrm{O}_{C}\right)$

Associativity ("semantic disunion"):

$\left(O_{A}\left(\ominus O_{B}\right) \quad \ominus O_{c}=O_{A}(\ominus)\left(O_{B}\left(\ominus O_{c}\right)\right.\right.$

$\underline{\underline{S}}$

- "semantic equality", binary operation.

Set of $A_{i}$ is semantically equal to set of $B_{i}$ if the following expression is true:

$$
\lim _{S} A_{i}=\lim _{S} B_{i}
$$

$\emptyset_{S}$ - "empty Sense Set".

Any No-Sense Set (Object No-Sense Set) is empty Sense Set. 
const

- "semantic constant".

For example, the following expression

$$
\lim _{S}\left(S_{f}\left(\phi_{N}\right)\right)=\text { const }
$$

means

$$
\operatorname{diff}_{(0)}^{\mathrm{n}}\left[S_{f}\left(\boldsymbol{S}_{N}\right)\right]_{M}=\underset{(\ominus)}{\operatorname{niff}}\left[S_{f}\left(\boldsymbol{S}_{N}\right)\right]_{M}=(
$$

for any element of

$\$_{N}$

The equivalent form is

$$
S_{f}^{\operatorname{diff}(n)}\left(\phi_{N}\right)=0
$$

\

- "attribute complement", binary operation

The attribute complement of ${ }^{\delta_{\llcorner}}\left(O_{B(L)}\right)$ in ${ }^{\delta_{k}}\left(O_{A(K)}\right)$ is the No-Sense Set ${ }^{\delta_{M}}\left(O_{C(M)}\right)$ of all elements that are members of ${ }^{\delta_{k}}\left(O_{A(K)}\right)$ but not members of ${ }^{\phi_{L}}\left(O_{B(L)}\right)$ :

$$
\not_{K} \backslash_{A} \not_{L}=\Phi_{M}\left(O_{A(K)} \backslash O_{B(L)}=O_{C(M)}\right)
$$

$\bar{\emptyset}_{S}$ - "empty set"

The empty set is a set that includes neither zero object nor No-Sense Set:

$$
\bar{\emptyset}_{S}:=" \prime\{\}
$$




$$
\begin{aligned}
& \underset{\mathrm{s}}{\in} \\
& \text { - "sense membership sign", binary operation } \\
& a_{1} \underset{\mathrm{S}}{\in \Phi_{\mathrm{N}}} \\
& S=\left\{\bigcirc_{\mathrm{K}} \odot \$_{\mathrm{N}}\right\}, N \geq K, a_{1}-\text { element of } \$_{\mathrm{N}}
\end{aligned}
$$

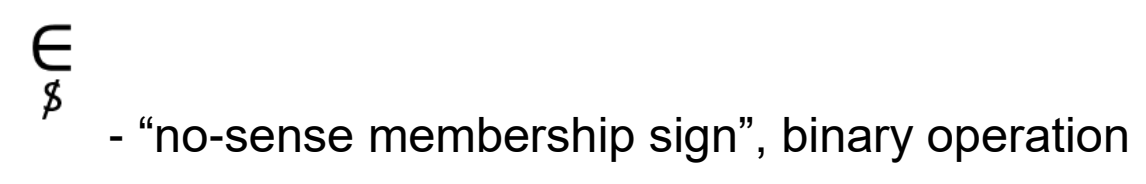

$\underset{S}{\notin}$

- "sense no-membership sign", binary operation

$a_{1} \notin \$_{\mathrm{N}}$

where $a_{1}$ is not an element of ${ }^{\phi_{N}}$ for which the following condition is met:

$$
S=\left\{\bigcirc_{\mathrm{K}} \Subset \phi_{\mathrm{N}}\right\}, N \geq K
$$

$$
\notin
$$

- "no-sense no-membership sign", binary operation

$$
a_{1} \notin \phi_{N}
$$

where $a_{1}$ is not an element of ${ }^{\Phi_{N}}$ which is not a sense

sequence

!

- "semantic complement", binary operation

The semantic complement of $S_{M}$ in $S_{N}$ is the Sense Set $S_{K}$ of all elements that are members of ${ }^{\delta_{N}}$ but not members of ${ }^{\delta_{m}}$ : 


$$
S_{N} \backslash_{\mathrm{s}} S_{M}=S_{K}, \text { where } a_{k} \underset{\mathrm{s}}{\in \not_{N}}, a_{k} \underset{\mathrm{s}}{\notin} \not_{\mathrm{M}}, S_{M} \stackrel{\mathrm{S}}{\not} S_{K}
$$

$\stackrel{\mathrm{S}}{\rightarrow}$ - "semantic implication", binary operation

If the expression (condition, relation) A exists, then the expression (condition, relation) $\mathrm{B}$ associated with $\mathrm{A}$ by one zero object also exists:

$$
A \stackrel{\mathrm{s}}{\rightarrow} B \text { where } A, B \underset{\mathrm{s}}{\in}(
$$

\section{Part 2.}

1. "ZO": (O), zero object

2. "NS": $\$$, No-Sense Set

3. "SI": $\odot$, semantic inclusion

4. "SE": $\odot$, semantic exclusion

5. "ISS": $S_{\not \subset}$, Incomplete Sense Set

6. "E": $\stackrel{\mathrm{E}}{|<=>|}$, equivalence

7. "NE": $\stackrel{|E=>|}{\mid E}$, no equivalence

8. "SL": $\lim _{S}$, sense limit

9. "SubS": $\operatorname{sub}(A)$, subset of $A$ set

10. "SU": () , semantic union

11. "SD": $(\ominus)$, semantic disunion

12. "SC": $\stackrel{\mathrm{S}}{\longrightarrow}$, semantic connection

13. "PN": $\stackrel{P N}{s}$, semantic punctured neighborhood 
14. "MoG": ${ }^{\S_{f}}$, module of gradient

15. "SEQ": , semantic equality 


\section{References}

[1] E. Mielberg, "Sense Theory. Part 1", 2018, https://vixra.org/pdf/1905.0105v1.pdf

[2] E. Mielberg, "Sense Space. Part 5", 2019, https://vixra.org/pdf/2012.0080v1.pdf

[3] E. Mielberg, "Sense Theory. Sense Function. Part 2", 2018, https://vixra.org/abs/1907.0527

[4] E. Mielberg, "Sense Theory. Antiderivative, Part 4", 2020, https://vixra.org/abs/2003.0583

[5] E. Mielberg, "Arllecta: A Decentralized Sense-To-Sense Network", 2019, https://vixra.org/abs/2002.0314

[6] E. Mielberg, "Sense Theory. Derivative, Part 3", 2019, https://vixra.org/abs/2003.0116

[7] E. Mielberg, "Sense Theory. Sense Diagrams, Part 6", 2020, https://vixra.org/abs/2010.0088

[8] E. Mielberg, "Sense Theory. Sense Series, Part 7", 2020, https://vixra.org/abs/2010.0081

[9] L. Dries, "Mathematical Logic", 2019,

https://faculty.math.illinois.edu/ vddries/main.pdf

[10] H. Schwichtenberg, "Mathematical Logic", 2003/2004,

https://faculty.math.illinois.edu/ vddries/main.pdf

[11] W. Rautenberg, "A Concise Introduction to Mathematical Logic", 2009, http://page.mi.fu-berlin.de/raut/logic3/announce.pdf

[12] I. Chiswell, W. Hodges, "Mathematical Logic", 2007, https://www.ibisc.univevry.fr/ belardinelli/Documents/Logique/Mathematical-LogicChiswellHodges.pdf 
[13] J. Keisler, K. Kunen, T. Millar, A. Miller, J. Robbin, "Mathematical Logic and Computability", 2006,

https://www.math.wisc.edu/ miller/res/book.pdf 